\title{
Evaluation of Mesiodistal Inclination of Permanent Maxillary and Mandibular First Molar in Different Angle's Molar Relation - A Cephalometric Study
}

\author{
Dweepika Garg, Santosh Kumar Goje
}

Department of Orthodontics and Dentofacial Orthopaedics, K. M. Shah Dental College and Hospital, Vadodara, Gujarat, India

Email for correspondence: dweepika_dg1@yahoo.co.in

\begin{abstract}
Context: Angle's molar relationship is the most widely used classification in spite of introduction of various other classifications. At the end of the treatment, the molars are finished in a Class I, II, or III molar relation. Achieving a proper cusp to fossa relation is necessary for attaining a balanced occlusion. Thus, it is important to know the ideal inclinations of the upper and lower first molars, thereby assisting in achieving stable relationship. Aims and Objectives: This study aims to evaluate mesiodistal inclination of permanent maxillary and mandibular first molars in Angle's Class I, II, and III molar relationships in relation to the occlusal plane. Materials and Methods: Lateral cephalograms of 16-30 years old individuals with Class I, II, and III molar relations were selected from the archives of the department of orthodontics. They were traced in Dolphin Imaging software, and the angle between the first molar and occlusal plane was calculated. Results: There was a statistically significant difference in the inclination of maxillary first molar in between Class I and Class III. No significant difference was noted in the inclination of maxillary first molar in case of Class I and Class II. In case of mandibular molars, there was statistically significant difference in case of Class I and Class II and between Class I and Class III. Conclusion: When the end molar relationship of a case is planned to be Class I, the upper molar should be at an angle of 84.12 to the occlusal plane. When the end molar relationship of a case is planned to be Class II, the upper molar should be 84.15 to the occlusal plane. When the end molar relationship of a case is planned to be Class III, the upper molar should be inclined at an angle of 87.07 to the occlusal plane. This helps in achieving an ideal cusp to fossa relationship, thereby stabilizing the occlusion.
\end{abstract}

Key words: Inclination, molar relation, molar tube, occlusal plane

\section{INTRODUCTION}

For orthodontists, incisors should not be the only concern. Molars, which are used as anchorage teeth, are also important, especially the first permanent

\begin{tabular}{|c|c|}
\hline Quick Response Code & Article Info: \\
\hline & doi: $10.5866 / 2018.10 .10074$ \\
\hline 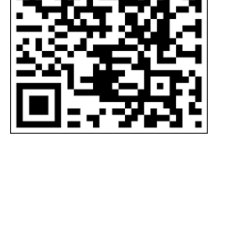 & $\begin{array}{l}\text { Received: } 26-04-2018 \\
\text { Revised: } 28-05-2018 \\
\text { Accepted: } 12-06-2018 \\
\text { Available Online: } 15-07-2018,2018 \\
\text { (www.nacd.in)@ NAD, } 2018 \text { - All rights } \\
\text { reserved }\end{array}$ \\
\hline
\end{tabular}

molars, the compensatory differences of which have crucial roles in growth, development, and anchorage control. ${ }^{[1]}$ In the specialty of orthodontics, the classification of malocclusion plays a very important role. It helps in diagnosis and treatment planning of malocclusion and to determine the magnitude of the problem. ${ }^{[2]}$ Second, classification facilitates communication between specialists.

Flavia et al. conducted a study to evaluate the mesiodistal root inclination of permanent incisors, canines, and first molar of Brazilian children with mixed dentition, using the horizontal reference line drawn on a panoramic radiograph. ${ }^{[3]}$ 
Several tools have been used for assessing the inclination of teeth: Cephalometric angular measurements, conveyor and compass on dental casts, etc. ${ }^{[4]}$ All teeth are arranged at an angle to the occlusal plane and each has an optimum inclination mesiodistally to best perform its individual and collective functions. ${ }^{[5]}$ Many clinicians have developed classification system for describing the malocclusion, namely Deway, ${ }^{[6]}$ Anderson, ${ }^{[7]}$ and Bennet. ${ }^{[8]}$ However, the most universally accepted classification system still in use today is $\mathrm{EH}$ angles method which was developed in 1889 and was based on the position of maxillary permanent first molar in the craniofacial anatomy. ${ }^{[2,9]}$

All teeth are essential, yet in function and influence: Some are of greater importance than others, the most important of all being maxillary first molar which is often called as: "Key to occlusion." ${ }^{\text {"2] }}$ Angle in 1906 published his article where he proposed the virtues of this tooth. ${ }^{[9]}$ George Risse suggested that maxillary first molar was the key to occlusion because of the following virtues: ${ }^{[2]}$

1. Largest teeth.

2. Firmest in their attachment.

3. Have a key location in the arch.

4. Broadest spread of root and widest base.

5. Occupy normal position in the arches far more often than teeth because they are first permanent teeth to erupt.

Crown inclination had been defined by Andrews in "The six keys to normal occlusion" based on his study of 120 adults with normal occlusion. ${ }^{[10]}$ Several orthodontists have found that the inclination of dentition can change appreciably according to certain factors and can exhibit regularities. Teeth could change their direction of eruption to compensate for positional changes of the jaws because the amount and direction of jaw growth showed considerable variability. ${ }^{[1]}$

Changes in inclination of the first permanent molars are inextricably linked to changes in Anchorage. Orthodontists have used various methods to make the correct changes in mesiodistal inclination of the first molars to influence anchorage preservation. Classic fixed appliances are used to put tip backs on the posterior teeth for resisting the forward-tipping trend of the molars.

Before starting the treatment, it is better to evaluate: (1) Molar inclination before treatment; (2) the benefits of the compensatory condition of the molars themselves for anchorage preservation; and (3) the natural pattern of differences in the axial inclination of the first molars in all types of malocclusions. ${ }^{[1]}$

Hong et al. evaluated the compensation trends of the inclination of first molar in Chinese population. Palatal and mandibular planes were used as reference planes. They concluded that the clinicians must avoid using a straight archwire in a 0 buccal tube on more distal-tipping first molars with regard to anchorage control..$^{[1]}$

During fixed orthodontic treatment, the buccal tube is placed in reference to the occlusal plane. Therefore, taking occlusal plane as reference would be better compared to mandibular plane or palatal plane so that buccal tube can be tipped mesially or distally, depending on the planned end relationship of molar. Hence, it will be better to know the mesiodistal inclination of molars in relation to occlusal plane which helps in accurate positioning of buccal tube.

Kamble et al. assessed 103 lateral cephalograms for positional variation of permanent maxillary first molar with infrazygomatic crest in skeletal Class I, II, and III cases. ${ }^{[2]}$ Mesiodistal root angulation alterations of the maxillary and mandibular incisors may significantly change the molar relationship. ${ }^{[3]}$

In Angle's Class I molar relation, mesiobuccal cusp of upper first permanent molar lies in the buccal groove of lower first permanent molar. Whereas in Class II molar relation, the mesiobuccal groove of upper first molar lies in the interdental area between the second PM and first molar. Thus, due to anatomic variations, there is change in the mesiodistal inclination of upper first molar in Class I, II, and III cases.

Kannabiran et al. concluded that tip and torque modifications are suggested for the fine finishing and easy retention of occlusion in Dravidians while using straight wire appliance. ${ }^{[5]}$ Hong et al. concluded that compensation of molar inclination varied among different classifications of sagittal malocclusion. ${ }^{[1]}$

After searching the literature databases such as PubMed, Medline, Ebsco, Scopus, and LILACS, till October 2017, with no restriction on language; very few studies 1,2 , and 3 are found which have evaluated the mesiodistal inclination of upper and lower first molar in Angles' Class I, II, and III cases. No study has been found which evaluated 
the mesiodistal inclination of upper and lower first molar in relation to the occlusal plane. Therefore, the present study has been taken up. The aim of the present study was to evaluate difference in the mesiodistal inclination of permanent maxillary and mandibular first molars in Angle's Class I, II, and III molar relationships in relation to the occlusal plane.

\section{MATERIALS AND METHODS}

The study was conducted in the Department of Orthodontics, K. M. Shah Dental College and Hospital, Piparia, Vadodara. Ethical approval was obtained from Sumandeep Vidyapeeth Institute of Ethical Clearance.

Based on values obtained from the study done by Kamble et al., ${ }^{[2]}$ the sample size was calculated. A sample size of 150 achieves $80 \%$ power to detect an effect size (W) of 0.25 using a 2 of freedom Chisquare test with a significance level (alpha) of 0.05 . Sample size N = Chi-Square/W $\mathrm{W}^{2}$; where $\mathrm{W}=0.25$; Chi-square $=9.4 ; \mathrm{DF}=2$. Hence, final sample size is 150 and sample per group is 50 .

Good quality, undamaged lateral cephalograms of Angles Class I, Class II, and Class III molar relation were included in the study. The exclusion criteria were the absence of permanent first molar or any tooth mesial or distal to it, lateral cephalograms showing bone loss around the first molar, any pathological lesion on the radiograph, lower anterior crowding, and cephalograms showing open bite.

Study models were assessed from the archives of pre-treatment orthodontic records of the patients. Study models with ideal posterior intercuspation were identified and their lateral cephalograms were included in the study. A total number of 50 lateral cephalograms of Class I, 50 Class II, and 50 Class III cases were recruited retrospectively based on the inclusion criteria.

The soft copies of the lateral cephalograms were digitized using Dolphin cephalometric software. Occlusal plane was traced by drawing a line passing through the intercuspation of upper and lower teeth. The long axis of the upper first molar was constructed from the apex of mesiobuccal root of the first molar and its mesiobuccal cusp using the software. The line was extended until it intersected the occlusal plane. Then, the superior inside angle was measured. Similarly, long axis of the lower first molar was constructed by joining the apex of mesiobuccal root with mesiobuccal cusp. The lines were extended until it intersects the occlusal plane. Then, the inferior inside angle was measured [Figures 1-6]. The data obtained were subjected for statistical analysis.

\section{RESULTS}

In patients with Class I molar relation, the mean inclination of maxillary molar is 84.12 and mean inclination of mandibular molar is 85.23. In patients with Class II molar relation, the mean inclination of maxillary molar is 84.15 and mean

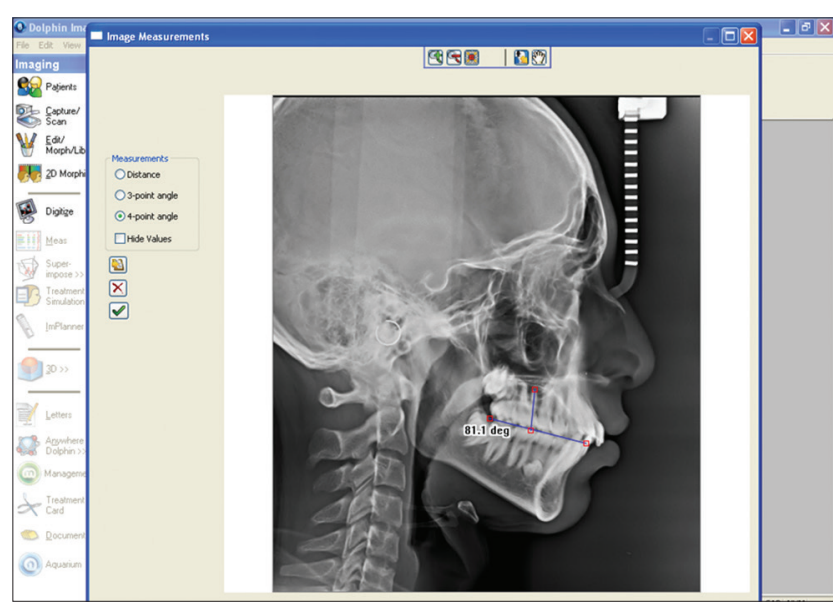

Figure 1: The long axis of the upper first molar was constructed from the apex of mesiobuccal root of the first molar and its mesiobuccal cusp using the software. The line was extended until it intersected the occlusal plane. Then, the superior inside angle was measured. A case of Class I molar relationship

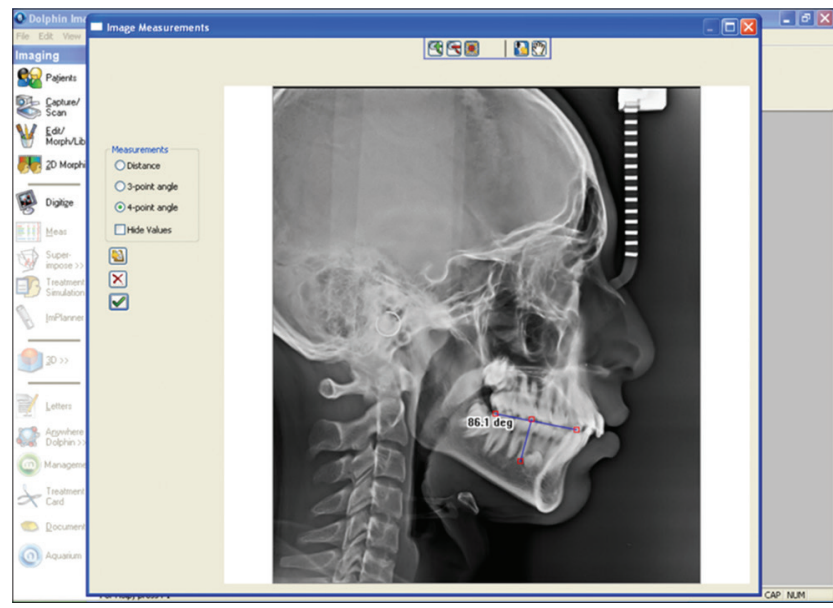

Figure 2: Long axis of the lower first molar was constructed by joining the apex of mesiobuccal root with mesiobuccal cusp. The lines were extended until it intersects the occlusal plane. Then, the inferior inside angle was measured. A case of Class I molar relation 


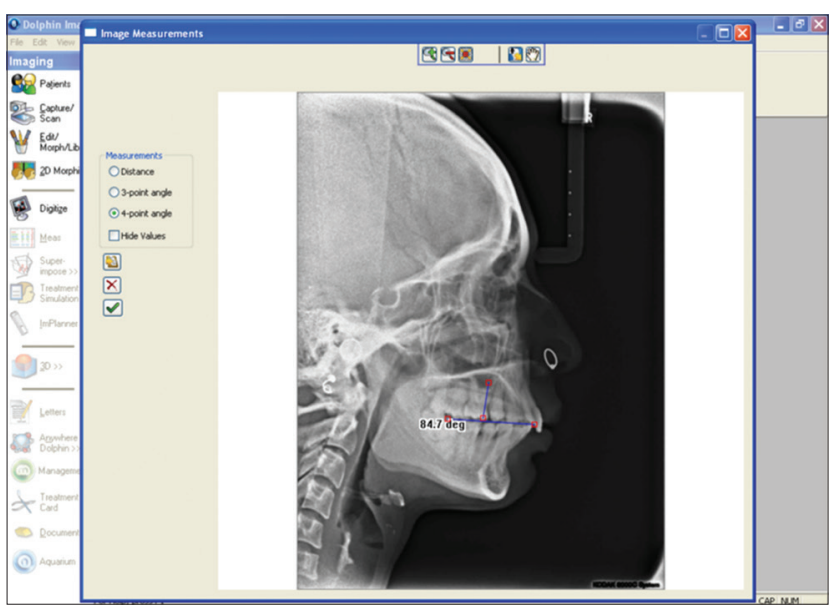

Figure 3: Inclination of upper molar measured in a case of Class II molar relation.

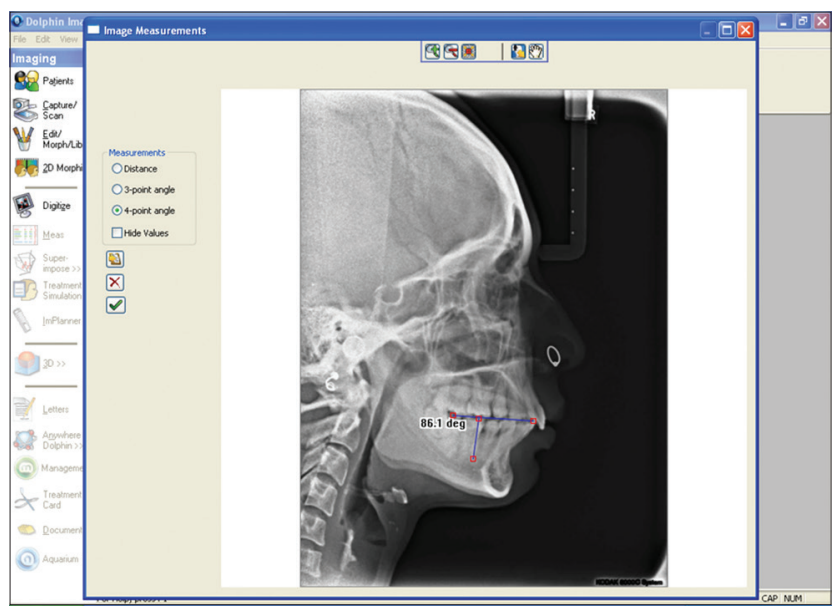

Figure 4: Inclination of lower molar measured in a Class II molar relation

inclination of mandibular molar is 81.71 . In patients with Class III molar relation, the mean inclination of maxillary molar is 87.07 and mean inclination of mandibular molar is 81.25 [Table 1 and 2].

Post hoc tests are used and multiple comparisons are done. When the inclination of maxillary molar is compared between Class I and Class II, $P$ value obtained is 0.999; showing the data obtained are not statistically significant. This indicates that there is no difference in the inclination of maxillary molar in cases of Class I and Class II molar relation. When the inclination of maxillary molar is compared between Class I and Class III, $P$ value obtained is 0.002; showing the data obtained are statistically significant. This indicates that there is significant difference in the inclination of maxillary molar in cases of Class I and Class III molar relation. When

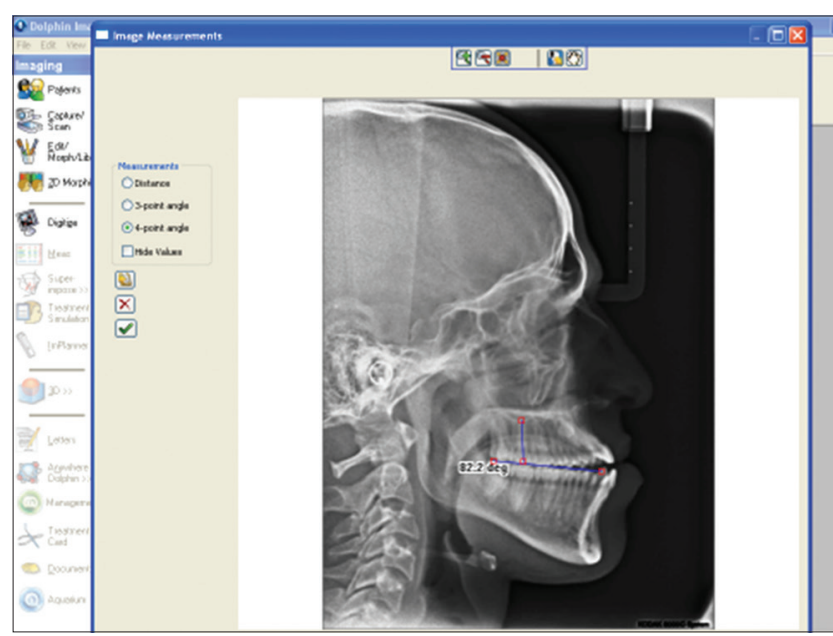

Figure 5: Inclination of upper molar measured in case of Class III molar relation

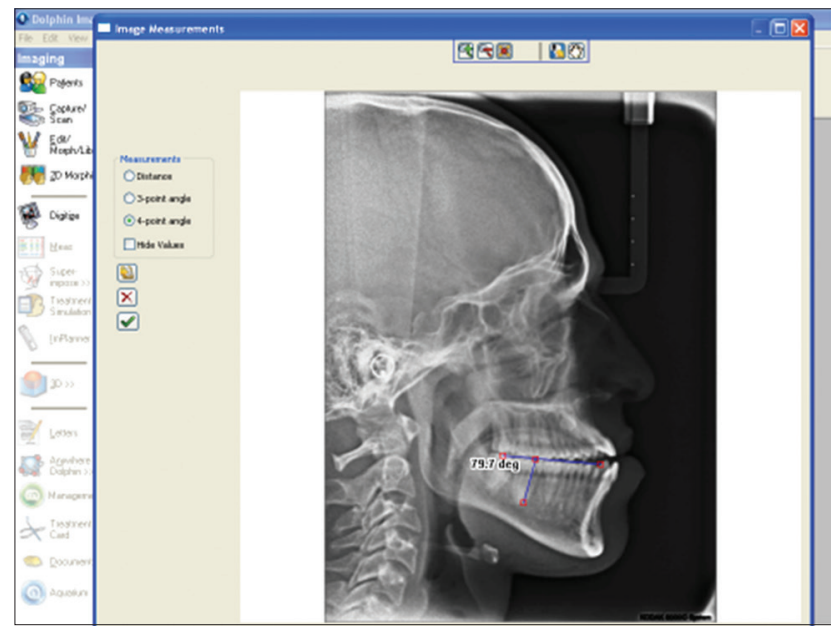

Figure 6: Inclination of lower molar measured in a Class III molar

the inclination of mandibular molar is compared between Class I and Class II, $P$ value obtained is 0.003; showing the data obtained are statistically significant. This indicates that there is significant difference in the inclination of mandibular molar in cases of Class I and Class II molar relation. When the inclination of mandibular molar is compared between Class I and Class III, $P$ value obtained is 0.001 ; showing the data obtained are statistically significant. This indicates that there is significant difference in the inclination of mandibular molar in cases of Class I and Class III molar relation [Table 3].

In Class I molar relation, the mean difference in the inclination of maxillary and mandibular first molar is 1.10. $P$ value obtained is 0.225 . This 


\begin{tabular}{|c|c|c|c|c|c|c|c|c|}
\hline \multirow[t]{2}{*}{ Group } & \multirow[t]{2}{*}{$\mathbf{n}$} & \multirow[t]{2}{*}{ Mean } & \multirow[t]{2}{*}{ SD } & \multirow[t]{2}{*}{ Std. error } & \multicolumn{2}{|c|}{$95 \%$ confidence interval for mean } & \multirow[t]{2}{*}{ Min. } & \multirow[t]{2}{*}{ Max. } \\
\hline & & & & & Lower bound & Upper bound & & \\
\hline \multicolumn{9}{|l|}{ Upper } \\
\hline I Molar & 50 & 84.12 & 3.62 & 0.51 & 83.09 & 85.15 & 75.90 & 90.00 \\
\hline II Molar & 50 & 84.15 & 4.82 & 0.68 & 82.78 & 85.52 & 72.00 & 92.40 \\
\hline III Molar & 50 & 87.07 & 4.24 & 0.60 & 85.86 & 88.27 & 71.40 & 92.40 \\
\hline Total & 150 & 85.11 & 4.45 & 0.36 & 84.39 & 85.83 & 71.40 & 92.40 \\
\hline \multicolumn{9}{|l|}{ Lower } \\
\hline I Molar & 50 & 85.23 & 4.80 & 0.68 & 83.86 & 86.59 & 65.50 & 95.00 \\
\hline II Molar & 50 & 81.71 & 5.05 & 0.71 & 80.27 & 83.14 & 64.90 & 92.00 \\
\hline III Molar & 50 & 81.25 & 5.68 & 0.80 & 79.64 & 82.86 & 65.50 & 90.50 \\
\hline Total & 150 & 82.73 & 5.45 & 0.45 & 81.85 & 83.61 & 64.90 & 95.00 \\
\hline
\end{tabular}

SD: Standard deviation

Table 2: ANOVA

\begin{tabular}{lccccc} 
Group & $\begin{array}{c}\text { Sum of } \\
\text { Squares }\end{array}$ & df & $\begin{array}{c}\text { Mean } \\
\text { Square }\end{array}$ & F & $\begin{array}{c}\text { ANOVA } \\
\text { P value }\end{array}$ \\
\hline Upper & & & & & \\
Between groups & 286.376 & 2 & 143.188 & 7.905 & 0.001 \\
Within groups & 2662.803 & 147 & 18.114 & & \\
Total & 2949.178 & 149 & & & \\
Lower & & & & & \\
Between groups & 472.949 & 2 & 236.474 & 8.786 & 0.0002 \\
Within groups & 3956.438 & 147 & 26.915 & & \\
Total & 4429.387 & 149 & & & \\
\hline
\end{tabular}

shows that there is no significant difference in the inclination of upper and lower molars in Class I cases. In Class II molar relation, the mean difference in the inclination of maxillary and mandibular first molar is 2.44. $P$ value obtained is 0.008 . This shows that there is statistically significant difference in the inclination of upper and lower first molars in Class II cases. In Class III molar relation, the mean difference in the inclination of maxillary and mandibular first molar is 5.81. $P$ value obtained is $<0.001$. This shows that there is statistically significant difference in the inclination of upper and lower first molars in Class III cases [Table 4].

\section{DISCUSSION}

The present study was carried out with the aim of evaluating the difference in the mesiodistal inclination of permanent maxillary and mandibular first molars in Angle's Class I, II, and III molar relationships in relation to the occlusal plane. The molar tube is placed in reference to the occlusal plane. Till now, no study had measured the inclination of molars in relation to the occlusal plane. Therefore, this study was taken up.

Lateral cephalograms of 16-30 years old individuals with Class I, II, and III molar relations were taken and were digitized using Dolphin cephalometric software. 4-point angle was taken. Only angular values were noted. No calibration was required.

All the values obtained were subjected to statistical analysis. Thus, the ideal inclination of upper and lower molar was identified. Hence, while ending a case in Class I, II, or III molar relationship, the inclination of maxillary and mandibular molar should be calculated in relation to occlusal plane. If it is not in the range of being ideal, then the molar tube can be tipped as per requirement to obtain the required inclination.

This will help in achieving proper inclination of molar at the end of the treatment. Thus, due to anatomical variations in the molar relations, its inclination varies in Class I, II, and III molar relationships. To achieve ideal inclination of the first molar after the treatment, proper positioning of molar tube is a necessity. Thus, by knowing the variations which needs to be done while bonding the first molar, it is possible to achieve good cusp to fossa relation, thereby stabilizing the occlusion. Hence, once the molar relation was decided in which the case has to end, the molar tube should be positioned accurately.

\section{CONCLUSION}

After appraising the inclination of molars, it can be concluded that 


\section{Table 3: Post hoc tests}

Multiple comparisons

\begin{tabular}{|c|c|c|c|c|c|}
\hline \multicolumn{6}{|l|}{ Tukey HSD } \\
\hline \multirow[t]{2}{*}{ Dependent variable } & \multirow[t]{2}{*}{ Mean difference (I-J) } & \multirow[t]{2}{*}{ Std. Error } & \multirow[t]{2}{*}{$P$ value } & \multicolumn{2}{|c|}{$95 \%$ confidence interval } \\
\hline & & & & Lower bound & Upper bound \\
\hline \multicolumn{6}{|l|}{ Upper } \\
\hline \multicolumn{6}{|l|}{ I Molar } \\
\hline II Molar & -0.026 & 0.851 & 0.999 & -2.04 & 1.99 \\
\hline III Molar & 2.944 & 0.851 & 0.002 & -4.96 & 0.93 \\
\hline \multicolumn{6}{|l|}{ II Molar } \\
\hline I Molar & 0.026 & 0.851 & 0.999 & 1.99 & 2.04 \\
\hline III Molar & 2.918 & 0.851 & 0.002 & -4.93 & -0.90 \\
\hline \multicolumn{6}{|l|}{ III Molar } \\
\hline I Molar & 2.944 & 0.851 & 0.002 & 0.93 & 4.96 \\
\hline II Molar & 2.918 & 0.851 & 0.002 & 0.90 & 4.93 \\
\hline \multicolumn{6}{|l|}{ Lower } \\
\hline \multicolumn{6}{|l|}{ I Molar } \\
\hline II Molar & 3.518 & 1.038 & 0.003 & 1.06 & 5.97 \\
\hline III Molar & 3.974 & 1.038 & 0.001 & 1.52 & 6.43 \\
\hline \multicolumn{6}{|l|}{ II Molar } \\
\hline I Molar & -3.518 & 1.038 & 0.003 & 5.97 & -1.06 \\
\hline III Molar & 0.456 & 1.038 & 0.899 & -2.00 & 2.91 \\
\hline \multicolumn{6}{|l|}{ III Molar } \\
\hline I Molar & 3.974 & 1.038 & 0.001 & 6.43 & -1.52 \\
\hline II Molar & -0.456 & 1.038 & 0.899 & -2.91 & 2.00 \\
\hline
\end{tabular}

\section{Table 4: Paired sample $T$-test}

\begin{tabular}{|c|c|c|c|c|c|c|}
\hline Group & Mean & $n$ & $\mathbf{S D}$ & Std. Error Mean & Mean difference & $P$ value \\
\hline \multicolumn{7}{|c|}{ Class-I molar } \\
\hline Upper & 84.12 & 50 & 3.62 & 0.512 & 1.10 & 0.225 \\
\hline Lower & 85.23 & 50 & 4.80 & 0.678 & & \\
\hline \multicolumn{7}{|c|}{ Class-II molar } \\
\hline Upper & 84.15 & 50 & 4.82 & 0.682 & -2.44 & 0.008 \\
\hline Lower & 81.71 & 50 & 5.05 & 0.715 & & \\
\hline \multicolumn{7}{|c|}{ Class-III molar } \\
\hline Upper & 87.07 & 50 & 4.24 & 0.600 & -5.81 & $<0.001$ \\
\hline Lower & 81.25 & 50 & 5.68 & 0.803 & & \\
\hline
\end{tabular}

SD: Standard deviation

1. When the end molar relationship of a case is planned to be Class I, the upper molar should be at an angle of 84.12 to the occlusal plane.

2. When the end molar relationship of a case is planned to be Class II, the upper molar should be 84.15 to the occlusal plane.
3. When the end molar relationship of a case is planned to be Class III, the upper molar should be inclined at an angle of 87.07 to the occlusal plane.

4. This helps in achieving an ideal cusp to fossa relationship, thereby stabilizing the occlusion. 


\section{REFERENCES}

1. Su H, Han B, Li S, Na B, Ma W, Xu TM, et al. Compensation trends of the angulation of first molars: Retrospective study of 1403 malocclusion cases. Int J Oral Sci 2014;6:175-81.

2. Kamble R, Hazarey A, Hazarey P, Singh J; Assessment of positional variation of maxillary permanent first molar with respect to the infrazygomatic crest(key ridge) in skeletal class I, II and III cases. W J Dent 2013;4:228-34.

3. Jesuino FA, Costa LR, Valladares-Neto J. Mesiodistal root angulation of permanent teeth in children with mixed dentition and normal occlusion. J Appl Oral Sci 2010;18:625- 9

4. Nouri M, Abdi AH, Farzan A, Mokhtarpour F, Baghban AA Measurement of the buccolingual inclination of teeth: Manual technique vs 3-dimensional software. Am J Orthod
Dentofacial Orthop 2014;146:522-9

5. Kannabiran P, Thirukonda GJ, Mahendra L. The crown angulations and inclinations in Dravidian population with normal occlusion. Indian J Dent Res 2012;23:53-8.

6. Dewey M. Classification of malocclusion. Int J Orthod 1915;1:133-47.

7. Anderson GM. Practical Orthodntics. $9^{\text {th }}$ ed. St.Louis: CV Mosby; 1960. p. 144-50.

8. Bennet NG. Report of the committee of orthodontic classification. Oral Health 1912;2:321-7.

9. Angle EH. Classification of malocclusion. Dent Cosmos 1899;41:248-64.

10. Andrews LF. The six keys to normal occlusion. Am J Orthod 1972;62:296-309. 